\title{
Pseudomonas and Neutrophil Products Modify Transferrin and Lactoferrin to Create Conditions That Favor Hydroxyl Radical Formation
}

\author{
Bradley E. Britigan ${ }^{* *}$ and Brian L. Edeker ${ }^{*}$ \\ * Research Service and Department of Internal Medicine, Veterans Administration Medical Center, and \\ ${ }^{\ddagger}$ Department of Internal Medicine, University of Iowa College of Medicine, Iowa City, Iowa 52242
}

\begin{abstract}
In vivo most extracellular iron is bound to transferrin or lactoferrin in such a way as to be unable to catalyze the formation of hydroxyl radical from superoxide $\left(\mathrm{O}_{2}^{-}\right)$and hydrogen peroxide $\left(\mathrm{H}_{2} \mathrm{O}_{2}\right)$. At sites of Pseudomonas aeruginosa infection bacterial and neutrophil products could possibly modify transferrin and/ or lactoferrin forming catalytic iron complexes. To examine this possibility, diferrictransferrin and diferriclactoferrin which had been incubated with pseudomonas elastase, pseudomonas alkaline protease, human neutrophil elastase, trypsin, or the myeloperoxidase product $\mathrm{HOCl}$ were added to a hypoxanthine/xanthine oxidase ${ }^{\circ} \mathrm{O}_{2}^{-} / \mathrm{H}_{2} \mathrm{O}_{2}$ generating system. Hydroxyl radical formation was only detected with pseudomonas elastase treated diferrictransferrin and, to a much lesser extent, diferriclactoferrin. This effect was enhanced by the combination of pseudomonas elastase with other proteases, most prominently neutrophil elastase. Addition of pseudomonas elastasetreated diferrictransferrin to stimulated neutrophils also resulted in hydroxyl radical generation. Incubation of pseudomonas elastase with transferrin which had been selectively iron loaded at either the $\mathrm{NH}_{2}$ - or $\mathrm{COOH}$-terminal binding site yielded iron chelates with similar efficacy for hydroxyl radical catalysis. Pseudomonas elastase and $\mathrm{HOCl}$ treatment also decreased the ability of apotransferrin to inhibit hydroxyl radical formation by a Fe-NTA supplemented hypoxanthine/xanthine oxidase system. However, apotransferrin could be protected from the effects of $\mathrm{HOCl}$ if bicarbonate anion was present during the incubation. Apolactoferrin inhibition of hydroxyl radical generation was unaffected by any of the four proteases or HOCl. Alteration of transferrin by enzymes and oxidants present at sites of pseudomonas and other bacterial infections may increase the potential for local hydroxyl radical generation thereby contributing to tissue injury. (J. Clin. Invest. 1991. 88:1092-1102.) Key words: iron • elastase • myeloperoxidase • alkaline protease $\bullet$ trypsin
\end{abstract}

\section{Introduction}

Sites of Pseudomonas aeruginosa and many other bacterial infections are notable for an intense infiltration of neutrophils which generate a variety of toxic oxygen species as an impor-

This work was presented in part at the Interscience Conference on Antimicrobial Agents and Chemotherapy, Atlanta, GA, 24 October 1990.

Address correspondence to Bradley E. Britigan, M.D., Dept. of Internal Medicine, University of Iowa, SW54, GH, Iowa City, IA 52242.

Received for publication 2 October 1990 and in revised form 4 June 1991.

The Journal of Clinical Investigation, Inc.

Volume 88, October 1991, 1092-1102 tant aspect of their antimicrobial armamentarium $(1,2)$. Membrane attachment of bacteria activates a unique membrane associated NADPH-dependent oxidase system which generates superoxide $\left(\mathrm{O}_{2}^{-}\right)^{1}$ and hydrogen peroxide $\left(\mathrm{H}_{2} \mathrm{O}_{2}\right)$, the latter via the spontaneous dismutation of ${ }^{\circ} \mathrm{O}_{2}^{-}(1,2)$. Interaction of $\mathrm{H}_{2} \mathrm{O}_{2}$ with myeloperoxidase (MPO) released from neutrophil cytoplasmic granules generates additional microbicidal oxidants such as hypochlorous acid $(\mathrm{HOCl})$ and chloramines $(3,4)$. Finally, in the presence of an exogenous iron catalyst, neutrophilderived ${ }^{\circ} \mathrm{O}_{2}^{-}$and $\mathrm{H}_{2} \mathrm{O}_{2}$ can also react to form the extremely toxic hydroxyl radical (5).

Neutrophil oxidant formation has been implicated as an important contributor to tissue injury at sites of inflammation (2). A variety of in vitro and in vivo studies have suggested that hydroxyl radical may be one of the reactive oxygen species involved (2). Because neutrophils do not possess an endogenous iron catalyst for hydroxyl radical generation (5-11), in order for this species to be produced in vivo, an exogenous catalytic iron complex must be present. In vivo almost all extracellular iron is tightly bound to host iron-binding proteins (12-14). Transferrin, a 76,000-80,000-D protein which is capable of binding two molecules of ferric iron, is the principal iron-binding protein in serum. It is also present in low concentrations at mucosal surfaces, but the related iron-binding protein lactoferrin provides the majority of the iron-chelating capacity at these locations (14). Although occasional reports to the contrary have appeared (15-17), it is generally believed that iron bound to either transferrin or lactoferrin is unable to participate as a hydroxyl radical catalyst (18-21). This feature of the iron-(lacto)transferrin complex may allow it to function as an inhibitor of oxidant-mediated tissue injury by decreasing the potential for the generation of hydroxyl radical $(22,23)$.

A variety of other neutrophil- and bacteria-derived products, particularly proteases, have been suggested to contribute to tissue injury at sites of $P$. aeruginosa infection (24-31). A number of these proteases have been reported to alter the physicochemical nature of transferrin and lactoferrin. Doring and colleagues (32) have shown that the $P$. aeruginosa secretory products pseudomonas elastase and pseudomonas alkaline protease cleave both transferrin and lactoferrin into smaller protein fragments. Pseudomonas elastase cleavage of diferrictransferrin enhances the removal of iron from transferrin by the pseudomonas siderophore pyoverdin (32). Neutrophil elastase, trypsin, chymotrypsin, and pepsin also appear to be able to cleave transferrin and lactoferrin (32-37). In addition, exposure of transferrin to oxidant products of the $\mathrm{MPO} / \mathrm{H}_{2} \mathrm{O}_{2}$ reaction (e.g., $\mathrm{HOCl}$ ) has been shown to interfere with the subsequent ability of the protein to bind ${ }^{59} \mathrm{Fe}$ without causing release of previously bound iron (38-42).

1. Abbreviations used in this paper: $\mathrm{MPO}$, myeloperoxidase; ${ }^{\circ} \mathrm{O}_{2}^{-}$, superoxide; TBA, thiobarbituric acid. 
The tertiary structure of transferrin and lactoferrin is likely important to their ability to bind ferric iron in a form incapable of acting as an hydroxyl radical catalyst. Therefore, the possibility that exposure of these iron-binding proteins to pseudomonas or neutrophil proteases, or $\mathrm{HOCl}$, could lead to generation of new iron chelates capable of catalyzing hydroxyl radical formation was examined. In the present communication, we report the results of these studies and discuss them in the context of potential mechanisms of inflammatory tissue injury with particular emphasis on that tissue injury associated with $P$. aeruginosa infection.

\section{Methods}

Reagents. Pseudomonas elastase and pseudomonas alkaline protease were kindly made available by Dr. Charles Cox, University of Iowa, Department of Microbiology, and Dr. Robert Fick, Jr., University of Iowa, Department of Internal Medicine. Human neutrophil elastase was purchased from Elastin Products, Owensville, MO. Trypsin, hypoxanthine, 2-deoxyribose, thiobarbituric acid, 5,5 dimethyl-1-pyrroline1-oxide (DMPO), $\alpha$-phenyl- $n$-tertbutyl-nitrone (PBN), CuZn superoxide dismutase (SOD), catalase, nitrilotriacetic acid (NTA), and diethylenetriaminepentaacetic acid (DTPA) were all obtained from Sigma Chemical Co., St. Louis, MO. Xanthine oxidase was purchased from Boehringer-Mannheim Biochemicals, Indianapolis, IN, dimethyl sulfoxide (DMSO) from Fisher Scientific Co., Fairlawn, NJ, and hypochlorous acid (HOCl) as $5 \%$ sodium hypochlorite (Regal Liquid Bleach) from Iowa Prison Industries, Anamosa, IA.

Iron-loading of lactoferrin and transferrin. Apotransferrin and apolactoferrin were purchased from Sigma Chemical Co. or CalbiochemBehring Corp., La Jolla, CA. In some cases diferric complexes of lactoferrin and transferrin were also obtained from the same commercial sources. Alternatively, apolactoferrin $(20 \mathrm{mg} / \mathrm{ml})$ or apotransferrin $(20$ $\mathrm{mg} / \mathrm{ml}$ ) were incubated in $200 \mathrm{mM} \mathrm{NH} \mathrm{HCO}_{3}$ (pH 8.2) containing sufficient ferric-NTA to achieve $70-80 \%$ saturation of the lactoferrin/ transferrin iron binding sites present. After $1 \mathrm{~h}$ at $37^{\circ} \mathrm{C}$, the preparations were placed in 12,000-14,000 mol wt membrane exclusion tubing (Spectropor; Spectrum Medical Industries, Inc., Los Angeles, CA) and dialyzed $24 \mathrm{~h}$ against 0.5 liters PBS with $1 \mathrm{mM} \mathrm{NaHCO}$ and $8 \mathrm{~g}$ chelex 100 beads (Bio-Rad Laboratories, Inc., Richmond, CA).

Transferrin in which the $\mathrm{NH}_{2}$ - or $\mathrm{COOH}$-terminal iron-binding site was selectively loaded was prepared using previously described methods $(43,44)$. In each case successful selective loading was confirmed by determining sample migration in a $6 \mathrm{M}$ urea gel (see below).

Protease cleavage of lactoferrin and transferrin. For experiments performed with iron-loaded transferrin or lactoferrin, the protein was loaded according to the above protocol. For experiments in which apolactoferrin or apotransferrin were employed, commercial preparations (Sigma Chemical Co. or Calbiochem-Behring Corp.) were used without further modification. Lactoferrin or transferrin mixtures $(20 \mathrm{mg} / \mathrm{ml})$ were incubated in the presence of pseudomonas elastase $(0.2-200 \mu \mathrm{g} /$ $\mathrm{ml})$, pseudomonas alkaline protease $(200 \mu \mathrm{g} / \mathrm{ml})$, neutrophil elastase $(200 \mu \mathrm{g} / \mathrm{ml})$, or trypsin $(50 \mu \mathrm{g} / \mathrm{ml})$. The standard duration for most experiments was $48-72 \mathrm{~h}$ at $37^{\circ} \mathrm{C}$. In some cases shorter durations of incubation with pseudomonas elastase were performed. Control samples were incubated in parallel in the absence of added proteases. In some cases, after protease incubation, samples were placed in 12,000 $14,000 \mathrm{~mol} \mathrm{wt}$ membrane exclusion tubing and dialyzed at $4^{\circ} \mathrm{C}$ for $3 \mathrm{~h}$ against 0.5 liters $\mathrm{PBS}$ containing $1 \mathrm{mM} \mathrm{NaHCO}$ and which had previously been treated with chelating resin (Sigma Chemical Co.) to remove adventitious metals. Extent of protease cleavage was assessed by SDS-PAGE (see below).

Hypochlorous acid treatment of lactoferrin and transferrin. Transferrin (apo or diferric) or lactoferrin (apo or diferric) prepared as above were incubated in $1 \mathrm{mM} \mathrm{NaHCO}$ containing $\mathrm{HOCl}$ at a 20-fold molar excess of transferrin or lactoferrin $(250 \mu \mathrm{M} \mathrm{HOCl}: 12.5 \mu \mathrm{M}$ transferrin or lactoferrin) for $30 \mathrm{~min}$ at $37^{\circ} \mathrm{C}$. In some cases, incubations with apotransferrin were performed in the absence of $\mathrm{NaHCO}_{3}$. The pH of buffer containing $\mathrm{NaHCO}_{3}$ was titrated with $\mathrm{HCl}$ so that it was identical with nonbicarbonate containing buffer ( $\mathrm{pH} 7.5$ ).

$S D S-P A G E$. Desired transferrin or lactoferrin preparations were solubilized by boiling in Laemmli solubilizing buffer (45) containing 2-mercaptoethanol (1\%) for $5 \mathrm{~min}$. Samples (7.5-12 $\mu \mathrm{g}$ protein/lane) were electrophoresed ( 150 volt hours) into a $10 \%$ polyacrylamide minigel after which the proteins were stained with silver according to the method of Wray et al. (46).

Urea gels. $6 \mathrm{M}$ ultrapure grade urea (Sigma Chemical Co.) was added to the standard $6 \%$ polyacrylamide gel preparation gel formulation ( $1.93 \mathrm{ml}$ polyacrylamide, $4.3 \mathrm{ml} 3 \mathrm{M}$ Tris [pH 8.92], $0.1 \mathrm{ml} 5 \%$ TEMED, and $0.24 \mathrm{ml} 1.5 \%$ ammonium persulfate) with $\mathrm{H}_{2} \mathrm{O}$ substituted for SDS. Transferrin or lactoferrin was mixed with $7 \mathrm{M}$ urea and 10:1 with glycerol. A small amount of bromophenol blue was sometimes added to monitor migration of the gel front. The running buffer used was $6 \mathrm{M}$ urea, $0.1 \mathrm{M}$ Tris, $0.01 \mathrm{M}$ boric acid, and $0.05 \mathrm{M}$ EDTA (47). Gels were run $5 \mathrm{~h}$ at $100 \mathrm{~V}$ (constant voltage) on ice, and then stained with Coomassie blue (48).

Formation of TBA-reactive 2-deoxyribose oxidation products. This assay was performed using a slight modification (49) of previously described methods $(9,50)$. Briefly, desired 1-ml reaction mixtures were prepared containing hypoxanthine $(2 \mathrm{mM})$ and 2-deoxyribose $(5 \mathrm{mM})$, $+/-$ exogenous iron chelates such that the final iron concentration was $25 \mu \mathrm{M}$. Xanthine oxidase $(15 \mathrm{mU} / \mathrm{ml})$ was then added and the reaction mixture incubated for $15 \mathrm{~min}$ at $37^{\circ} \mathrm{C}$ (rotating incubator). $0.5 \mathrm{ml}$ of $1 \%$ thiobarbituric acid (TBA) and $1 \mathrm{ml} \mathrm{6 \%}$ TCA was then added following which the mixture was heated $\left(100^{\circ} \mathrm{C} \times 10 \mathrm{~min}\right)$. The protein was then pelleted $(1,000 \mathrm{~g}, 5 \mathrm{~min})$ and the absorbance of the resulting supernatant at $532 \mathrm{~nm}$ determined using a model DU-30 spectrophotometer (Beckman Instruments, Inc., Irvine, CA).

Spin trapping. Spin trapping was performed using two previously developed methods $(5,11)$. Xanthine oxidase $(31 \mathrm{mU} / \mathrm{ml})$ was added to a solution containing hypoxanthine $(2 \mathrm{mM})$, DTPA $(0.1 \mathrm{mM})$, DMSO (140 mM), and DMPO (100 mM) or PBN (10 mM), +/- exogenous iron chelate $(0.1-0.2 \mathrm{mM})$. The reaction mixture was transferred to a flat quartz EPR cell and the EPR spectrum determined using a model E104A EPR spectrometer (Varian Associates, Palo Alto, CA), which was kindly made available by Dr. Harold Goff (University of Iowa, Department of Chemistry). In some cases SOD $(30 \mathrm{U} / \mathrm{ml})$ or catalase $(500 \mathrm{U} / \mathrm{ml})$ were also included in the reaction mixture. Unless otherwise indicated EPR spectrometer settings were: microwave power, $20 \mathrm{~mW}$; modulation amplitude, $1 \mathrm{G}$; time constant, $1 \mathrm{~s}$; modulation frequency, $100 \mathrm{kHz}$; sweep rate $12.5 \mathrm{G} / \mathrm{min}$; and receiver gain, 5.0 $\times 10^{4}$.

Statistical analysis. A paired Student's $t$ test was used for all statistical analyses. Results were considered significant at $P<0.05$. To enhance the effectiveness of presentation, in some cases data are expressed as a percentage of appropriate control. However, original (raw) data were used for all statistical comparisons.

\section{Results}

Protease cleavage of diferrictransferrin and diferriclactoferrin. The tertiary structures of lactoferrin and transferrin are likely important to their ability to bind iron in a form incapable of acting as a hydroxyl radical catalyst. Because previous studies (32-37) demonstrated the ability of several proteases to cleave these iron-binding proteins, the ability of the resulting protease-induced cleavage products to function as hydroxyl radical catalysts was assessed. Four proteases were targeted for study: pseudomonas elastase, pseudomonas alkaline protease, human neutrophil elastase, and trypsin. The two pseudomonas proteases were selected because they have previously been suggested as virulence factors for pseudomonas-associated tissue 
injury (24-27, 32). Neutrophil elastase was chosen as a model for phagocyte-derived serine proteases and because it has been linked to some forms of inflammatory tissue injury (51). Finally, trypsin was felt to be representative of local mucosal proteases.

First the relative ability of each protease to cleave diferrictransferrin and diferriclactoferrin was assessed. Diferrictransferrin or diferriclactoferrin were incubated with pseudomonas elastase, pseudomonas alkaline protease, neutrophil elastase, or trypsin for $48-72 \mathrm{~h}$ at $37^{\circ} \mathrm{C}$. The number and apparent molecular weight of the cleavage products differed with each of the proteases employed (Figs. 1-4). Similarly the extent of cleavage of the native protein also varied (Figs. 1-4). For diferrictransferrin the extent of cleavage was pseudomonas elastase $>$ trypsin $>$ neutrophil elastase $>$ alkaline protease (Figs. 1-4). For diferriclactoferrin the hierarchy was similar (Figs. 1-4). Relative to diferrictransferrin, diferriclactoferrin appeared to be more resistant to proteolysis by trypsin (Fig. 4).

Ability of protease-cleaved diferrictransferrin and diferriclactoferrin to catalyze hydroxyl radical production. Having confirmed the ability of the various proteases to cleave the two iron-binding proteins, the ability of the protease-treated diferrictransferrin and diferriclactoferrin complexes to function as a hydroxyl radical catalyst was examined. The oxidation of hypoxanthine by xanthine oxidase generates ${ }^{\circ} \mathrm{O}_{2}^{-}$and $\mathrm{H}_{2} \mathrm{O}_{2}$ but not hydroxyl radical $(52,53)$. Thus, detection of hydroxyl radical production by this reaction in the presence of an iron chelate is an indication of the ability of that chelate to function as a hydroxyl radical catalyst. Accordingly, diferrictransferrin, diferriclactoferrin, or these proteins which had been previously incubated with one of the four proteases described above were added to a mixture of hypoxanthine and 2-deoxyribose. Xanthine oxidase was then added to initiate ${ }^{\circ} \mathrm{O}_{2}^{-}$and $\mathrm{H}_{2} \mathrm{O}_{2}$ generation after which production of hydroxyl radical was quantitated by measuring the formation of TBA-reactive 2-deoxyribose oxidation products (Table I). Evidence for hydroxyl radical production was apparent with the addition of pseudomonas elastase-treated diferrictransferrin to the hypoxanthine/xanthine oxidase system (Table I). This was not related to release of free iron from the protein as dialysis of the pseudomonas elastase cleaved transferrin in $12,000-14,000 \mathrm{~mol} \mathrm{wt}$ exclusion tubing against chelating resin failed to reduce the

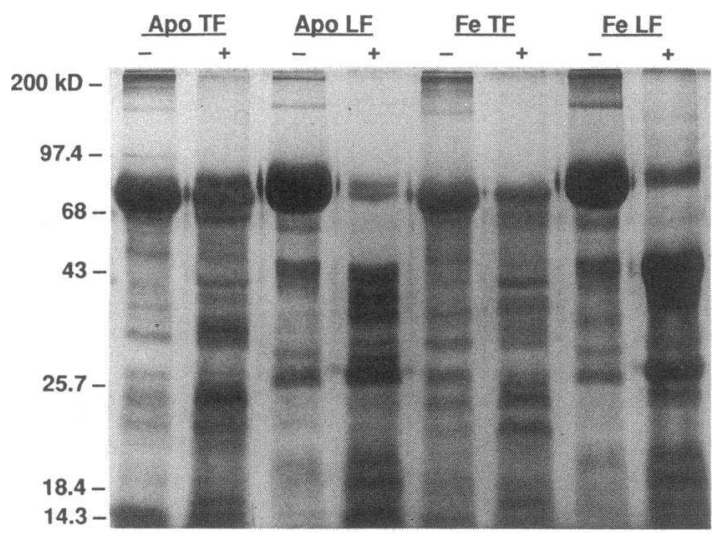

Figure 1. SDS-PAGE of apotransferrin (apoTF), apolactoferrin (apoLF), diferrictransferrin (FeTF), and diferriclactoferrin (FeLF) incubated $48 \mathrm{~h}$ in the absence (-) or presence $(+)$ of $200 \mu \mathrm{g} / \mathrm{ml} \mathrm{pseu-}$ domonas elastase.

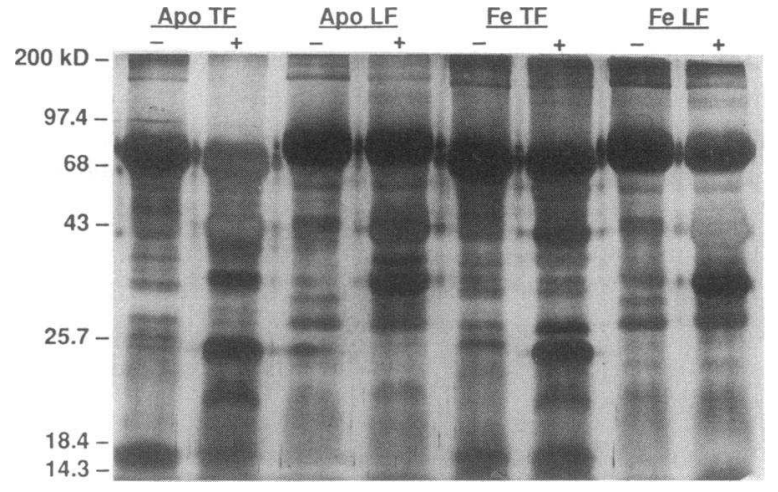

Figure 2. SDS-PAGE of apotransferrin (apoTF), apolactoferrin (apoLF), diferrictransferrin (FeTF), and diferriclactoferrin (FeLF) incubated $48 \mathrm{~h}$ in the absence $(-)$ or presence $(+)$ of $200 \mu \mathrm{g} / \mathrm{ml} \mathrm{hu}-$ man neutrophil elastase.

amount of hydroxyl radical generated with its subsequent addition to the hypoxanthine/xanthine oxidase system (not shown). A small but statistically significant amount of hydroxyl radical was also observed with pseudomonas elastasetreated diferriclactoferrin (Table I). Hydroxyl radical catalysis was not detected with use of untreated diferrictransferrin or diferriclactoferrin (not shown), or with either of these two compounds which had been preincubated with pseudomonas alkaline protease, neutrophil elastase, or trypsin (Table I). Addition of pseudomonas elastase to the hypoxanthine/xanthine oxidase system in the absence of diferrictransferrin or diferriclactoferrin did not suggest that the protease itself functioned as a hydroxyl radical catalyst (data not shown). Similarly, use of apotransferrin which had been cleaved by pseudomonas elastase did not result in generation of hydroxyl radical confirming that iron associated with the transferrin was the source of hydroxyl radical catalysis.

Confirmation of the ability of pseudomonas elastase-induced cleavage products to act as a hydroxyl radical catalyst. Although the formation of TBA-reactive deoxyribose oxidation products appears to be relatively specific for hydroxyl radical, additional confirmation that pseudomonas elastase cleavage of diferrictransferrin yields catalytic iron chelates was sought using spin trapping techniques. Free radicals by defini-

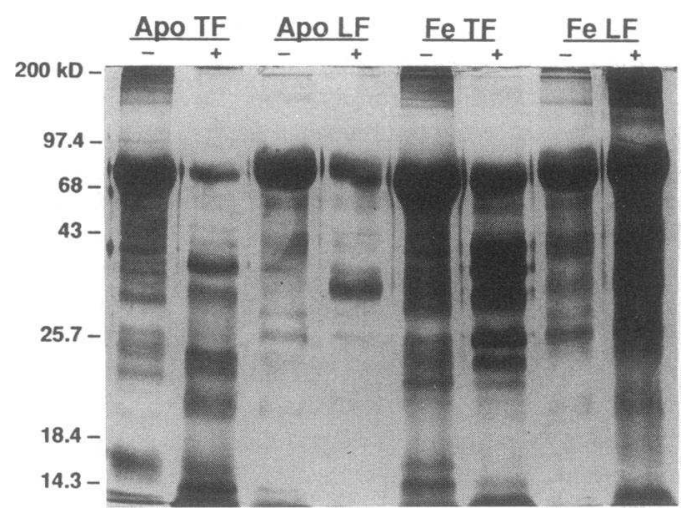

Figure 3. SDS-PAGE of apotransferrin (apoTF), apolactoferrin (apoLF), diferrictransferrin (FeTF), and diferriclactoferrin (FeLF) incubated $48 \mathrm{~h}$ in the absence $(-)$ or presence $(+)$ of $200 \mu \mathrm{g} / \mathrm{ml} \mathrm{pseu-}$ domonas alkaline protease. 


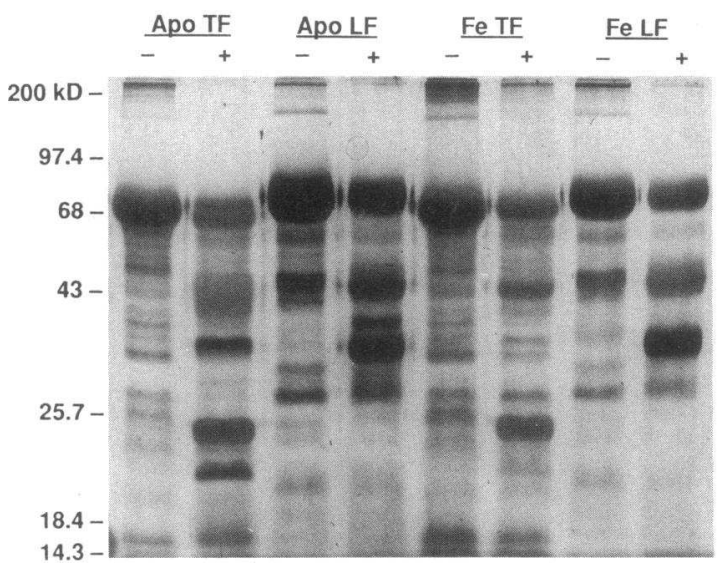

Figure 4. SDS-PAGE of apotransferrin (apoTF), apolactoferrin (apoLF), diferrictransferrin (FeTF), and diferriclactoferrin (FeLF) incubated $48 \mathrm{~h}$ in the absence $(-)$ or presence $(+)$ of $50 \mu \mathrm{g} / \mathrm{ml}$ trypsin.

tion contain an unpaired electron which generates a small magnetic moment detectable by EPR spectrometry. Because of their short half-life and reasons inherent to EPR spectrometry most oxygen-centered radicals such as ${ }^{\circ} \mathrm{O}_{2}^{-}$and hydroxyl radical are not detectable at physiologic temperatures using this technique. However, ${ }^{\circ} \mathrm{O}_{2}^{-}$and hydroxyl radical will react with

Table I. Generation of Catalytic Iron Chelates by Treatment of Diferrictransferrin and Diferriclactoferrin with Different Proteases

\begin{tabular}{llc}
\hline & \multicolumn{2}{c}{ Absorbance (532 nm) } \\
\cline { 2 - 3 } & \multicolumn{1}{c}{ Fe-TF } & Fe-LF \\
\hline PAE [200] & $0.128 \pm 0.018(24)^{8}$ & $0.034 \pm 0.010(7)^{*}$ \\
{$[20] \times 48 \mathrm{~h}$} & $0.073 \pm 0.005(6)^{8}$ & $0.048 \pm 0.019(3)$ \\
{$[20] \times 24 \mathrm{~h}$} & $0.119 \pm 0.022(5)^{\ddagger}$ & $\mathrm{ND}$ \\
{$[20] \times 12 \mathrm{~h}$} & $0.048 \pm 0.016(5)^{*}$ & $\mathrm{ND}$ \\
{$[20] \times 6 \mathrm{~h}$} & $0.034 \pm 0.009(5)^{*}$ & $\mathrm{ND}$ \\
{$[2]$} & $0.028 \pm 0.006(3)$ & $0.034 \pm 0.024(3)$ \\
{$[0.2]$} & $0.012 \pm 0.005(3)$ & $0.028 \pm 0.003(3)$ \\
PAP & $0.003 \pm 0.003(3)$ & $0.005 \pm 0.005(3)$ \\
HNE & $0.016 \pm 0.007(9)$ & $0.001 \pm 0.001(3)$ \\
Trypsin & $0.015 \pm 0.011(3)$ & $0.009 \pm 0.003(3)$ \\
\end{tabular}

Formation of TBA-reactive 2-deoxyribose oxidation products (532 $\mathrm{nm}$ absorbance) reflecting formation of hydroxyl radical by reaction mixtures composed of hypoxanthine, xanthine oxidase, and diferrictransferrin (Fe-TF) or diferriclactoferrin (Fe-LF), which were untreated or had been previously exposed to pseudomonas elastase (PAE), pseudomonas alkaline protease (PAP), human neutrophil elastase (HNE), or trypsin. The absorbance expressed represents the mean of results obtained $\left(A_{532}\right)$ with the addition to the hypoxanthine/xanthine oxidase system of protease-treated diferrictransferrin or diferriclactoferrin less the $\mathbf{A}_{532}$ obtained with a paired control (untreated) iron chelate. Numbers in parenthesis after each set of data indicate the number of separate experiments performed. Incubations were for 48-72 $\mathrm{h}$ except where noted with exposure of diferric transferrin to $20 \mu \mathrm{g} / \mathrm{ml}$ PAE. Shown in brackets are the concentrations of PAE $(\mu \mathrm{g} / \mathrm{ml})$ used in specific sets of experiments. The final concentration of iron present was $25 \mu \mathrm{M}$. Statistically different from the hypoxanthine/xanthine oxidase diferric(lacto)transferrin control, ${ }^{*} P$ $<0.05,{ }^{\ddagger} P<0.01$, and ${ }^{\S} P<0.0001$, respectively. some nitrone compounds, termed spin traps, to generate stable nitroxide free radicals readily detectable by EPR. The unique hyperfine splitting pattern of the EPR spectrum of these species allows identification of the original free radical species. We have previously employed spin trapping systems for the detection of hydroxyl radical in biologic systems $(5,11)$. In the presence of the spin trap DMPO and DMSO hydroxyl radical is manifested as the methyl radical spin adduct of DMPO, $\mathrm{DMPO} / \mathrm{CH}_{3}(5)$. When hydroxyl radical is generated in a system containing DMSO and a different spin trap, PBN, the $\mathrm{PBN} / \mathrm{OCH}_{3}$ spin adduct results (11).

Consistent with earlier studies (18-22), when diferrictransferrin or diferriclactoferrin was added to a hypoxanthine/ xanthine oxidase system containing DMSO and either DMPO or PBN, no evidence of hydroxyl radical generation was detected (Figs. 5-7). Addition of diferrictransferrin which had been previously exposed to pseudomonas elastase to the hypoxanthine/xanthine oxidase system resulted in the generation of spin adducts of DMPO and PBN expected from the formation of hydroxyl radical, DMPO/ $\mathrm{CH}_{3}$ and $\mathrm{PBN} / \mathrm{OCH}_{3}$, respectively (Figs. 5 and 6). Detection of these spin adducts was appropriately inhibited $80-100 \%$ by the inclusion of catalase (Figs. 5 and 6) or SOD (not shown) in the reaction mixture. Consistent with the results of the deoxyribose oxidation assay, addition of pseudomonas elastase cleaved diferriclactoferrin to the hypoxanthine/xanthine oxidase reaction yielded spin trap evidence of a small amount of hydroxyl radical formation (Fig. 7).

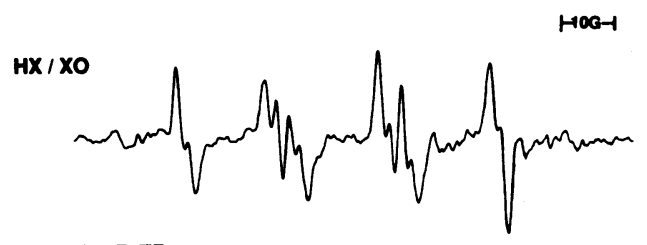

HX/XO+FETF

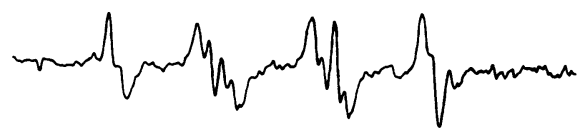

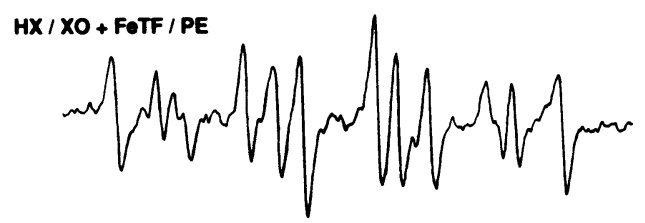

$\mathrm{HX} / \mathrm{XO}+\mathrm{FOTF} / \mathrm{PE}+\mathrm{Cat}$

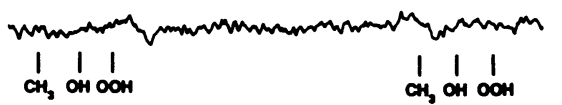

Figure 5. EPR spectra representative of three to five separate experiments obtained with the addition of xanthine oxidase to DMPO, DMSO, DTPA, and hypoxanthine (HX/XO) as well as the same reaction mixture supplemented with diferrictransferrin $(0.1 \mathrm{mM} \mathrm{Fe}$, $+\mathrm{FeTF}$ ) or the same concentration of diferrictransferrin previously preincubated with $200 \mu \mathrm{g} / \mathrm{ml}$ pseudomonas elastase (+ FeTF/PE). Also shown is the spectrum obtained with the pseudomonas elastase cleaved diferrictransferrin if catalase $(500 \mathrm{U} / \mathrm{ml})$ was also included (+ FeTF/PE, + Cat). High- and low-field peaks corresponding to the spin adducts DMPO/ $/{ }^{\circ} \mathrm{CH}_{3}, \mathrm{DMPO} /{ }^{\circ} \mathrm{OH}$, and DMPO/ ${ }^{\circ} \mathrm{OOH}$ are designated $\mathrm{CH}_{3}, \mathrm{OH}$, and $\mathrm{OOH}$, respectively. 

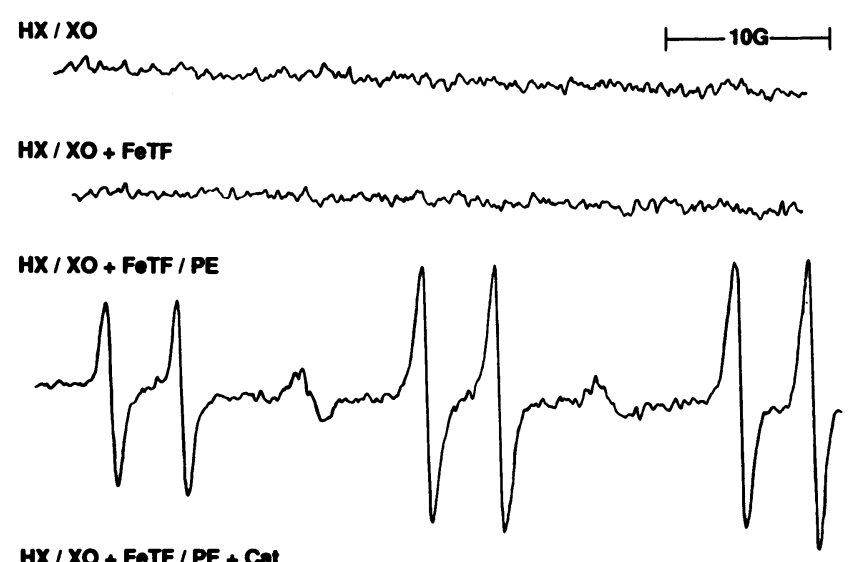

$H X / X O+F E T F / P E+C a t$

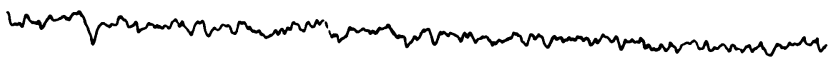

Figure 6. EPR spectra representative of three separate experiments obtained after the addition of xanthine oxidase to PBN, DMSO, DTPA, and hypoxanthine (HX/XO) as well as the same reaction mixture supplemented with diferrictransferrin $(0.1 \mathrm{mM} \mathrm{Fe},+\mathrm{FeTF})$ or the same concentration of diferrictransferrin previously cleaved with pseudomonas elastase (+ FeTF/PE). Also shown is the effect of catalase $(500 \mathrm{U} / \mathrm{ml})$ on the FeTF/PE-induced spectrum (FeTF/PE + Cat). The spectrum observed with FeTF/PE is that of $\mathrm{PBN} /{ }^{\circ} \mathrm{OCH}_{3}$.

Effect of the pseudomonas elastase concentration and the combination of different proteases on the generation of a hydroxyl radical catalyst from diferrictransferrin and diferriclactoferrin. There exists considerable discrepancy in the literature as to the concentration of pseudomonas elastase which might be encountered in vivo $(26,54-59)$. To obtain greater insight into the potential biologic relevance of the above observations we varied the concentration of pseudomonas elastase to which diferrictransferrin and diferriclactoferrin were exposed before its addition to the hypoxanthine/xanthine oxidase system. As shown in Table I, evidence of hydroxyl radical catalysis was detectable after diferrictransferrin incubation with concentrations of pseudomonas elastase as low as $2 \mu \mathrm{g} / \mathrm{ml}$. In contrast to these results, statistically significant hydroxyl radical generation was not detected using diferriclactoferrin exposed to concentrations of pseudomonas elastase $\leq 20 \mu \mathrm{g} / \mathrm{ml}$ (Table I).

In addition to assessing the effect of pseudomonas elastase concentration, the effect of the duration of diferrictransferrin exposure to pseudomonas elastase exposure on the generation of catalytic iron chelates was also examined. Diferrictransferrin incubated with $20 \mu \mathrm{g} / \mathrm{ml}$ pseudomonas elastase for as short as 6 $\mathrm{h}$ was able to catalyze hydroxyl radical formation (Table I). The magnitude of hydroxyl radical production was increased with the duration of incubation (Table I). Incubations beyond $24 \mathrm{~h}$ did not appear to increase the overall yield of catalytic iron chelate(s).

As discussed earlier sites of pseudomonas infection likely contain a variety of different proteases. Accordingly, the impact of a multiple protease mixture on diferrictransferrin and diferriclactoferrin was examined. Diferrictransferrin and diferriclactoferrin were incubated simultaneously in the presence of pseudomonas elastase, pseudomonas alkaline protease, human neutrophil elastase, and trypsin for 48-72 $\mathrm{h}$. This reaction mixture was then added to the hypoxanthine/xanthine oxidase system and magnitude of hydroxyl radical formation assessed
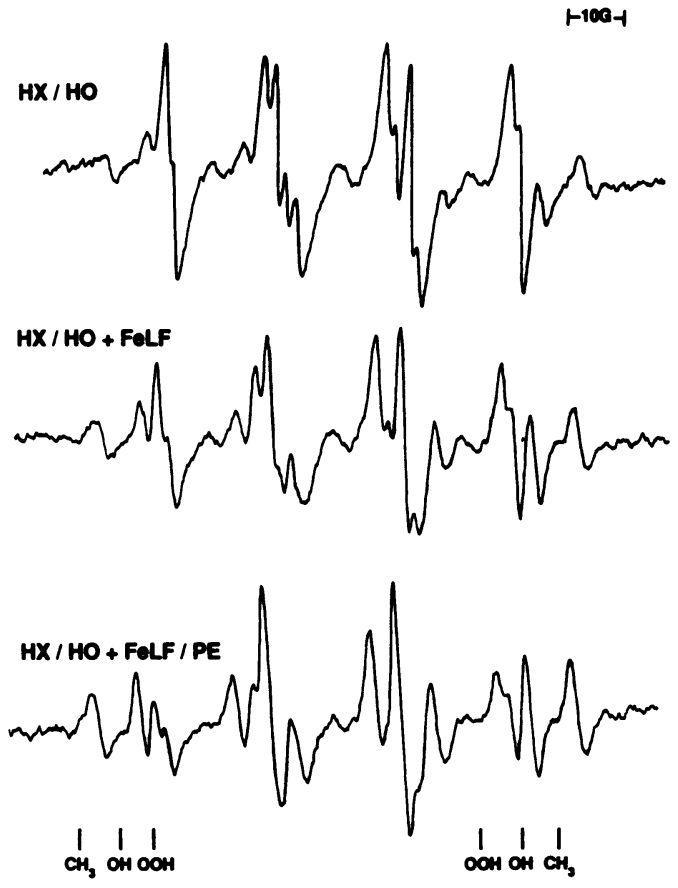

Figure 7. (A) EPR spectra obtained after the addition of xanthine oxidase to DMPO, DMSO, DTPA, and hypoxanthine (HX/XO) as well as the same reaction mixture supplemented with diferriclactoferrin $(0.1 \mathrm{mM} \mathrm{Fe},+\mathrm{FeLF})$ or the same concentration of diferriclactoferrin previously cleaved with pseudomonas elastase (+ FeLF/PE). Highand low-field peaks corresponding to the spin adducts DMPO/ $/{ }^{\circ} \mathrm{CH}_{3}$, DMPO/ ${ }^{\circ} \mathrm{OH}$, and DMPO/ ${ }^{\circ} \mathrm{OOH}$ are designated $\mathrm{CH}_{3}, \mathrm{OH}$, and $\mathrm{OOH}$, respectively. Note the small increase in $\mathrm{DMPO} /{ }^{\circ} \mathrm{CH}_{3}$ peak amplitude observed with FeLF/PE relative to the other two conditions.

by the deoxyribose oxidation assay. As shown in Table II, in the case of diferrictransferrin incubation in the presence of the four proteases enhanced the resulting formation of hydroxyl radical to a level almost five times that observed with incubation with pseudomonas elastase alone. This combination, however, had no effect on diferriclactoferrin (Table II). To assess which of the three proteases was responsible for enhancing the effect of pseudomonas elastase incubations of diferrictransferrin were performed with pseudomonas elastase paired with only one of the other three proteases. The combination of pseudomonas elastase with human neutrophil elastase appeared to account for much, but not all, of the enhanced effect of the protease combination noted above (Table II). Furthermore, as can be ascertained by comparing results in Tables I and II, neutrophil elastase markedly enhanced the ability of pseudomonas elastase to induce catalytic iron chelates at all concentrations of pseudomonas elastase studied $(0.2-20 \mu \mathrm{g} / \mathrm{ml})$. In contrast, exposure of diferrictransferrin to the combination of $20 \mu \mathrm{g} / \mathrm{ml}$ pseudomonas elastase and pseudomonas alkaline protease or trypsin yielded hydroxyl radical production which was somewhat less than that induced by pseudomonas elastase alone (Table II), although the decrease was not statistically significant.

Is the ability of pseudomonas elastase to generate catalytic iron chelates from diferrictransferrin restricted to only one binding site? Because transferrin has two iron-binding sites it seemed possible that the iron chelate resulting from pseudomonas elastase cleavage of diferrictransferrin was generated 
Table II. Generation of Catalytic Iron Chelates by Treatment of Diferrictransferrin and Diferriclactoferrin with Combinations of Proteases

\begin{tabular}{llc}
\hline & \multicolumn{2}{c}{ Absorbance (532 nm) } \\
\cline { 2 - 3 } & \multicolumn{1}{c}{ Fe-TF } & Fe-LF \\
\hline All proteases & $0.338 \pm 0.028(3)$ & $-0.020 \pm 0.011$ \\
PAE [20] + PAP & $0.016 \pm 0.025(3)$ & ND \\
PAE [20] + Trypsin & $0.033 \pm 0.006(3)$ & ND \\
PAE [20] + HNE & $0.183 \pm 0.022(3)$ & ND \\
PAE [2] + HNE & $0.291 \pm 0.037(3)$ & ND \\
PAE [0.2] + HNE & $0.157 \pm 0.052(3)$ & ND \\
\hline
\end{tabular}

Formation of TBA-reactive 2-deoxyribose oxidation products (532 $\mathrm{nm}$ absorbance) reflecting formation of hydroxyl radical by reaction mixtures composed of hypoxanthine, xanthine oxidase, and diferrictransferrin (Fe-TF) or diferriclactoferrin (Fe-LF), which had been previously exposed to combinations of pseudomonas elastase (PAE), pseudomonas alkaline protease (PAP), human neutrophil elastase (HNE), or trypsin for 48-72 h. The absorbance expressed represents the mean of results obtained $\left(A_{532}\right)$ with the addition to the hypoxanthine/xanthine oxidase system of protease-treated diferrictransferrin or diferriclactoferrin less the $\mathbf{A}_{532}$ obtained with a paired control (untreated) iron chelate. Numbers in parenthesis after each set of data indicate the number of separate experiments performed and numbers in brackets indicate the concentration of PAE $(\mu \mathrm{g} / \mathrm{ml})$ employed. The final concentration of iron present was $25 \mu \mathrm{M}$. Only diferrictransferrin treated with the four protease combination or the combination of pseudomonas elastase and neutrophil elastase demonstrated a statistically significant increase in hydroxyl radical formation relative to results obtained with exposure of the iron chelate to the same concentration of pseudomonas elastase alone, $P<0.05$.

from iron bound to only one binding site. Clarification of this possibility has clear biologic relevance since, except under rare circumstances, transferrin does not approach full saturation in vivo and the two binding sites are loaded in a nonrandom manner (60-62). Consequently, transferrin was selectively loaded at either the $\mathrm{COOH}$-terminal or $\mathrm{NH}_{2}$-terminal iron-binding site. Transferrin containing iron at either of these two binding sites was then incubated in the presence or absence of pseudomonas elastase for $48 \mathrm{~h}$. The fact that the iron remained associated with the binding site of interest was confirmed by noting the difference in migration of the $\mathrm{COOH}$-terminal and $\mathrm{NH}_{2}$-terminal loaded preparations on a $6 \mathrm{M}$ urea gel (47) at the end of the 48-h incubation period (Fig. 8). These transferrin prepara-

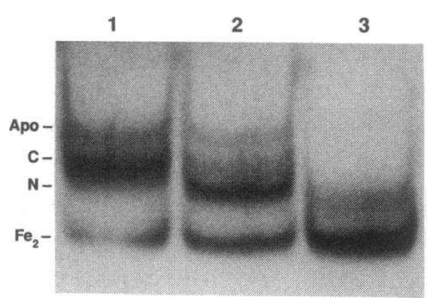

\footnotetext{
Figure 8. $6 \mathrm{M}$ urea gel stained with Coomassie blue of transferrin loaded with iron as detailed in Methods so as to selectively load the $\mathrm{COOH}$ - (lane 1), $\mathrm{NH}_{2}$ (lane 2) or both iron binding sites (lane 3).
}

Locations of bands corresponding to apo, $\mathrm{NH}_{2}$-terminal, $\mathrm{COOH}$-terminal and diferrictransferrin are designated. These gels were always obtained at the termination of the 48-72 $\mathrm{h}$ incubation procedure to assure that the iron was still associated with the same iron-binding site at the termination of the experiment. tions were then added to the hypoxanthine/xanthine oxidase system and hydroxyl radical production quantitated as formation of TBA-reactive deoxyribose oxidation products. Regardless of whether $\mathrm{COOH}$-terminal or $\mathrm{NH}_{2}$-terminal loaded transferrin was employed, pseudomonas elastase treatment resulted in the formation of hydroxyl radical catalysts of similar potency. Addition of sufficient $\mathrm{NH}_{2}$ - or $\mathrm{COOH}$-terminal loaded transferrin to generate a final iron concentration of $25 \mu \mathrm{M}$ yielded $\mathrm{A}_{532}$ of $0.224 \pm 0.029(n=5 ; P<0.05)$ and $0.228 \pm 0.021$ $(n=5 ; P<0.01)$, respectively. This is nearly identical to the value obtained with pseudomonas elastase cleaved diferrictransferrin at a similar iron concentration $\left(\mathrm{A}_{532}=0.214\right.$ $\pm 0.022, n=23$ ).

Exposure of diferrictransferrin and diferriclactoferrin to hypochlorous acid. In addition to bacteria- and neutrophil-derived proteases, at sites of inflammation transferrin and lactoferrin are likely exposed to MPO-derived oxidants. Several groups of investigators have examined the impact of products of the reaction between MPO and $\mathrm{H}_{2} \mathrm{O}_{2}$ such as $\mathrm{HOCl}$ on the iron-binding properties of transferrin and lactoferrin (38-42). These studies suggested that such exposure did not result in the release of ferric iron bound to either of these iron-binding proteins. However, the possibility that $\mathrm{HOCl}$ treatment of transferrin or lactoferrin could alter the potential for iron bound to these proteins to function as catalysts for hydroxyl radical formation was not addressed. Accordingly, diferrictransferrin or diferriclactoferrin were incubated in the presence of a 20 -fold molar excess of $\mathrm{HOCl}$ for $30 \mathrm{~min}$. These $\mathrm{HOCl}$-treated proteins were then added to a solution of hypoxanthine and 2-deoxyribose after which xanthine oxidase was added and formation of hydroxyl radical assessed as production of TBA-reactive deoxyribose oxidation products. Regardless of whether transferrin or lactoferrin were employed, no evidence of hydroxyl radical formation was detected. In comparison to results with untreated controls, $\mathrm{A}_{532}$ increased negligibly, a mean of $0.003(n=4)$ and $0.000(n=3)$ with HOCl-treated diferrictransferrin and diferriclactoferrin, respectively $(P>0.05)$. To be certain that $\mathrm{HOCl}$ did not effect either the ability of xanthine oxidase to generate ${ }^{\circ} \mathrm{O}_{2}^{-} / \mathrm{H}_{2} \mathrm{O}_{2}$ or the assay to detect hydroxyl radical production, a solution containing $\mathrm{HOCl}$ and a concentration of BSA identical to that of transferrin or lactoferrin present in the above experiments was added to the hypoxanthine/xanthine oxidase system which had been supplemented with the catalytic iron chelate ferricnitrilotriacetic acid. A similar magnitude of deoxyribose oxidation product formation was detected relative to a system containing BSA but no $\mathrm{HOCl}$ (not shown). These results suggest that $\mathrm{HOCl}$ treatment does not result in the generation of iron chelates of transferrin or lactoferrin which are able to act as hydroxyl radical catalysts.

Generation of hydroxyl radical by pseudomonas elastase cleaved diferrictransferrin in the presence of stimulated neutrophils. The above results with pseudomonas elastase cleavage products of diferrictransferrin suggests the possibility that they could contribute to tissue injury at sites of pseudomonas infection by catalyzing the formation of hydroxyl radical from ${ }^{\circ} \mathrm{O}_{2}^{-}$ and $\mathrm{H}_{2} \mathrm{O}_{2}$ generated by local neutrophils. However, as we have previously shown $(63,64)$ the release of lactoferrin during the neutrophil respiratory burst can decrease the likelihood of hydroxyl radical formation by chelating exogenous forms of iron in a noncatalytic form. To provide further insight into the likelihood of diferrictransferrin cleavage products contributing to in vivo tissue injury, formation of hydroxyl radical was measured 
after stimulation of human neutrophils with PMA in the presence of diferrictransferrin which had been previously cleaved with pseudomonas elastase. As assessed with the deoxyribose oxidation assay, hydroxyl radical was generated in the presence of pseudomonas elastase-treated diferrictransferrin $\left(\delta A_{532}\right.$ $=0.075 \pm 0.010, n=7, P<0.05)$ but not with untreated diferrictransferrin.

Protease and hypochlorous acid treatment of apotransferrin and apolactoferrin. Based on the work of other investigators (33-41), it seemed possible that the iron-binding properties of transferrin and lactoferrin might be more susceptible to protease or $\mathrm{HOCl}$ modification if the iron-binding proteins were exposed to these agents before their association with iron. Consequently, we incubated apotransferrin or apolactoferrin in the presence of pseudomonas elastase, pseudomonas alkaline protease, human neutrophil elastase, trypsin; or $\mathrm{HOCl}$ under the same conditions as the earlier experiments with diferric chelates (Figs. 1-4). In general, the relative susceptibility of diferrictransferrin and diferriclactoferrin to each of the proteases was similar to that noted with the apo forms of the molecules (Figs. 1-4). However, the nature and relative proportion of cleavage products generated by most proteases varied with use of apo as compared to diferric forms of transferrin or lactoferrin (Figs. 1-4). Most notably, both apotransferrin and apolactoferrin were cleaved to a much greater extent than the diferric forms by pseudomonas alkaline protease (Fig. 3). Apotransferrin was similarly more susceptible to neutrophil elastase than its diferric counterpart (Fig. 2). Finally, apolactoferrin appeared to be more resistant than apotransferrin to cleavage by trypsin and neutrophil elastase (Figs. 2 and 4).

The functional consequence of exposure of apotransferrin and apolactoferrin to each of the proteases was then examined by assessing the ability of the various protease cleavage products to inhibit iron catalyzed hydroxyl radical production. Each reaction product was added to a mixture of hypoxanthine, Fe-NTA, and deoxyribose. The resulting ability of the system to generate hydroxyl radical upon the addition of xanthine oxidase was then determined relative to the hypoxanthine/xanthine oxidase system in the presence of the untreated iron-binding proteins or in their absence. Pretreatment of apotransferrin with $\mathrm{HOCl}$ or pseudomonas elastase (20 or $200 \mu \mathrm{g} /$ $\mathrm{ml}$ ) but not the other proteases interfered with the ability of the compound to inhibit formation of hydroxyl radical (Table III). Analogous to results with diferrictransferrin, the combination of pseudomonas elastase with the other three proteases or just human neutrophil elastase (but not other combinations) enhanced the effect of pseudomonas elastase (Table III). Somewhat surprisingly, the deleterious effect of $\mathrm{HOCl}$ was not noted if bicarbonate anion $(1 \mathrm{mM})$ was present during the period of $\mathrm{HOCl}$ exposure (Table III). The final $\mathrm{pH}$ under both conditions was adjusted to 7.5 , eliminating that factor as the explanation for the observation. In contrast to the results with apotransferrin, the ability of apolactoferrin to inhibit formation of hydroxyl radical was not altered by exposure to any of the four proteases (alone or in combination) or $\mathrm{HOCl}$ (Table III).

\section{Discussion}

Recent evidence suggests that proteases likely present at sites of pseudomonas infection could potentially alter the iron chelating properties of transferrin and lactoferrin (32-37), proteins which may play an important role in decreasing the likelihood
Table III. Effect of Protease and HOCl Treatment on the Ability of Apo-Transferrin and Apo-Lactoferrin to Prevent Iron-catalyzed Hydroxyl Radical Formation

\begin{tabular}{lcc}
\hline & \multicolumn{2}{c}{$\begin{array}{c}\text { Percentage of control } \\
\text { hydroxyl radical generation }\end{array}$} \\
\cline { 2 - 3 } & ApoTF & ApoLF \\
\hline No treatment & $7(10)$ & $5(10)$ \\
PAE [200] & $36(4)$ & $5(3)$ \\
{$[20]$} & $40(7)$ & $7(7)$ \\
{$[2]$} & $5(5)$ & ND \\
{$[0.2]$} & $6(5)$ & ND \\
PAP & $3(4)$ & $5(3)$ \\
HNE & $10(4)$ & $6(3)$ \\
Trypsin & $9(4)$ & $7(3)$ \\
All proteases & $57(3)$ & $2(3)$ \\
PAE [20] + PAP & $21(3)$ & $2(3)$ \\
PAE [20] + Trypsin & $50(5)$ & $5(3)$ \\
PAE [20] + HNE & $63(4)$ & $6(3)$ \\
HOCl & $66(4)$ & $7(3)$ \\
HOCl/HCO & $8(3)$ & ND \\
\end{tabular}

Median inhibition of hydroxyl radical formation by the reaction of hypoxanthine and xanthine oxidase in the presence of Fe-NTA (10 $\mu \mathrm{M}$ ) with the inclusion of $1 \mathrm{mg} / \mathrm{ml}$ apotransferrin (ApoTF) or apolactoferrin (ApoLF) which had been treated previously with pseudomonas elastase (PAE), pseudomonas alkaline protease (PAP, $200 \mu \mathrm{g} /$ $\mathrm{ml})$, human neutrophil elastase (HNE, $200 \mu \mathrm{g} / \mathrm{ml})$, trypsin $(50 \mu \mathrm{g} / \mathrm{ml})$, or $\mathrm{HOCl}$. Concentration of PAE used $(\mu \mathrm{g} / \mathrm{ml})$ is shown in brackets. In the case of $\mathrm{HOCl}$ results are shown for exposure to $\mathrm{HOCl}$ in the presence or absence of $1 \mathrm{mM}$ bicarbonate anion. In each case data are expressed as the percentage of formation of TBA-reactive deoxyribose oxidation products $\left(A_{532}\right)$ detected relative to that obtained on the same day with the hypoxanthine/xanthine oxidase/Fe-NTA mixture in the absence of apotransferrin or apolactoferrin-derived proteins. Treatment of apotransferrin with pseudomonas elastase (20 or $200 \mu \mathrm{g} / \mathrm{ml}$ ) or with $\mathrm{HOCl}$ (in the absence of bicarbonate) resulted in a significant loss of the protein's ability to inhibit hydroxyl radical generation $(P<0.01)$. No other treatment with a single protease yielded statistically significant loss of apotransferrin inhibitory activity. The combination of the four proteases or HNE and PAE enhanced the effect observed with PAE-treated apotransferrin $(P<0.05)$.

of in vivo formation of hydroxyl radical $(22,23,63,64)$. Consequently, we assessed the impact of in vitro exposure of lactoferrin and transferrin to four different proteases (pseudomonas elastase, pseudomonas alkaline protease, human neutrophil elastase, and trypsin) on the potential for generation of the highly cytotoxic hydroxyl radical. Initial studies used the reaction of hypoxanthine and xanthine oxidase was used as a convenient model for neutrophil oxidant formation to avoid potential confounding influences of the proteases on various aspects of neutrophil function.

Incubation with any of the above proteases alone or in combination resulted in the cleavage of transferrin or lactoferrin into multiple subunits. Consistent with a previous report of its high resistance to protease cleavage (33) we found that human lactoferrin exhibited a greater overall resistance to proteolysis than transferrin. The number and apparent molecular weight of these cleavage products varied with the iron-binding protein and protease examined and are in general agreement with the 
work of other investigators (32-37). However, in contrast to our results (Fig. 1), Doring et al. (32) stated that no cleavage products above $14,000 \mathrm{D}$ were detected with the incubation of pseudomonas elastase with transferrin. The difference may relate to the fact that in the studies of Doring and colleagues (32) the ratio of pseudomonas elastase to transferrin was 30 -fold higher than in our studies. Finally, others have noted that relative to their iron loaded form, apolactoferrin and apotransferrin may be more resistant to protease digestion $(33,37)$. We did not find this to be the case, but the marked differences in experimental conditions used among the studies make comparisons difficult.

In the present work, only the products of the interaction of pseudomonas elastase and diferrictransferrin resulted in the generation of large amounts of hydroxyl radical when added to the hypoxanthine/xanthine oxidase ${ }^{\circ} \mathrm{O}_{2}^{-} / \mathrm{H}_{2} \mathrm{O}_{2}$ generating system. Pseudomonas elastase-cleaved diferrictransferrin was also able to catalyze formation of hydroxyl radical when a more physiologically relevant source of ${ }^{\circ} \mathrm{O}_{2}^{-} / \mathrm{H}_{2} \mathrm{O}_{2}$, stimulated neutrophils, was substituted for the hypoxanthine/xanthine oxidase system.

There did not appear to be a clear correlation between the magnitude of protease-induced cleavage and generation of catalytic iron chelates. For example, diferrictransferrin was extensively cleaved by both pseudomonas and neutrophil elastase. Yet only the pseudomonas elastase diferrictransferrin cleavage products catalyzed hydroxyl radical formation. As demonstrated by experiments in which transferrin was selectively loaded at only one iron-binding site, pseudomonas elastase apparently cleaves the transferrin molecule in such a way that iron bound to either the $\mathrm{COOH}$ - or $\mathrm{NH}_{2}$-terminal binding site becomes equally capable of acting as a hydroxyl radical catalyst. Dialysis of the pseudomonas elastase-cleaved transferrin indicated that these results were not related to simple release of bound iron. In addition, autoradiography of ${ }^{59} \mathrm{Fe}$-transferrin exposed to pseudomonas elastase has revealed multiple lower molecular weight fragments which have ${ }^{59} \mathrm{Fe}$ associated with them (Britigan, B. E., B. L. Edeker, and M. L. McCormick, unpublished observation). Although pseudomonas elastase cleavage of diferriclactoferrin also yielded products with some capacity to act as a hydroxyl radical catalyst, this required high concentrations of pseudomonas elastase. Even then, the magnitude of hydroxyl radical generated was quite small and therefore may not have biologic significance.

Wide variations have been reported in the concentration of enzymatically active pseudomonas elastase present at sites of infection with this organism. Most of this work has centered on studies of airway secretions obtained from patients with cystic fibrosis whose airways were infected/colonized with $P$. aeruginosa. Fick et al. detected pseudomonas elastase activity of $\sim 2 \mu \mathrm{g} / \mathrm{ml}$ in bronchoalveolar lavage samples from such patients (54). Taking into account the 100 -fold dilution such samples are usually felt to represent (65) this would represent a concentration of pseudomonas elastase in the airway of nearly $200 \mu \mathrm{g} / \mathrm{ml}$, close to the maximal concentration used in our study. Similarly, significant levels of pseudomonas elastase activity were reported by Bruce et al. (55). In contrast much lower or absent levels of pseudomonas elastase activity were detected in a similar group of patients in studies by others $(26,56-58)$. These groups have suggested that pseudomonas elastase may not be active in such individuals due to the presence in the airway of pseudomonas elastase-specific immunoglobulins which develop over time in such patients. However, as pointed out by Bruce et al. (55), these studies also vary in the way in which samples were collected and processed.

To our knowledge there is no data on levels of pseudomonas elastase present at sites of human pseudomonas infection in conditions other than cystic fibrosis. Using a rat model system in which $P$. aeruginosa was injected into a "granuloma pouch," Doring et al. (59) reported pseudomonas elastase and alkaline protease concentrations in inflammatory exudate of 0-185 and 0-742 $\mathrm{ng} / \mathrm{ml}$, respectively. These contrasted levels in in vitro culture supernatant using similar concentrations of organism where concentrations were in some cases 100-1,000fold higher than those measured in vivo. This difference was found to be in part explainable by a rapid clearance of active enzyme from the exudate fluid suggesting that the rate of elastase and alkaline protease production by the organism in vivo was considerably greater than would be predicted by steadystate levels. In addition, neither this study nor the ones mentioned above optimally address what the local concentration of enzyme might be in close proximity to sites where organisms are adherent to host cells as would be expected to occur in pulmonary airways.

In vivo sites of $P$. aeruginosa infection are characterized by the presence of multiple proteases. When, diferrictransferrin was simultaneously incubated with multiple proteases the generation of catalytic iron chelates was enhanced relative to that observed with pseudomonas elastase alone. Human neutrophil elastase although inactive alone enhanced the effect of pseudomonas elastase. This was associated with increased proteolysis observed with SDS-PAGE (not shown). In the presence of human neutrophil elastase the concentration of pseudomonas elastase required for the generation of catalytic iron chelates was reduced markedly to that which would be likely achievable under in vivo conditions. These results are in accordance with suggestions by other investigators $(26,66)$ that these two elastases could enhance the pathogenic potential of one another. Because the combination of pseudomonas elastase and neutrophil elastase were still not as effective in generating catalytic iron chelates as the combination of four proteases it is likely that the actions of multiple mucosal proteases could synergistically enhance the breakdown of transferrin in vivo.

In contrast to the results with human neutrophil elastase, there was a suggestion that both trypsin and pseudomonas alkaline protease may decrease the effectiveness of pseudomonas elastase in the generation of catalytic iron chelates from diferrictransferrin. Such an observation could result from inactivation of pseudomonas elastase by the other protease or potentially modification of transferrin cleavage resulting in products which retain their ability to bind iron in a noncatalytic form. However, because these decreases did not reach statistical significance and the results of experiments using a combination of all four proteases showed the greatest enhancement of catalytic iron formation, the above observation is probably of only marginal importance.

Similar to the results obtained with iron-loaded forms of transferrin, exposure of apotransferrin to pseudomonas elastase, but not the other three proteases, decreased its ability to inhibit hydroxyl radical generation by the hypoxanthine/ xanthine oxidase/Fe-NTA system which depends on the ability of apotransferrin to bind iron in a non-catalytic form. This could be due to either a loss of the overall ability of the pseudomonas elastase-induced transferrin cleavage products to bind 
iron or an alteration in the redox properties of bound iron. As noted with diferrictransferrin, the effect of pseudomonas elastase was enhanced if human neutrophil elastase and to a lesser extent trypsin was also present during the incubation. Analogous to results with diferriclactoferrin, exposure to either of the four proteases (or their combinations) did not appear to have any significant impact on the ability of apolactoferrin to inhibit hydroxyl radical formation by the same system. These results also did not appear to be explainable solely on the basis of the magnitude of proteolysis observed with each of the four proteases.

These data suggest that pseudomonas elastase may be somewhat unique among proteases in its ability to generate ferric complexes from transferrins which are capable of acting as hydroxyl radical catalysts or interfere with the subsequent ability of apo forms of these proteins to bind microenvironmental iron in a noncatalytic form. Lactoferrin, however, appears to be much more resistant than transferrin to such modification by pseudomonas elastase. These data are very consistent with the work of Doring et al. (32) which demonstrated that pseudomonas elastase treatment of iron-loaded transferrin but not lactoferrin allowed subsequent chelation of that iron by the pseudomonas siderophore pyoverdin. In the same study (32), exposure of transferrin or lactoferrin to either neutrophil elastase or pseudomonas alkaline protease did not result in enhanced removal of iron from these proteins by pyoverdin.

Transferrin and lactoferrin have been reported to be susceptible to oxidation by products of the reaction of MPO and $\mathrm{H}_{2} \mathrm{O}_{2}$ such as $\mathrm{HOCl}(38-42)$. Nevertheless exposure of either diferrictransferrin or diferriclactoferrin to $\mathrm{HOCl}$ did not generate iron chelates capable of catalyzing hydroxyl radical generation. However, exposure of apotransferrin but not apolactoferrin to $\mathrm{HOCl}$ decreased the ability of that iron-binding protein to inhibit hydroxyl radical formation by the hypoxanthine/xanthine oxidase/Fe-NTA system. These data support and extend the recent work of both Clark and Pearson (39) and Winterbourn and Malloy (38). The former investigators reported (39) that exposure of transferrin to stimulated neutrophils or a cellfree MPO/ $\mathrm{H}_{2} \mathrm{O}_{2}$ system in the presence of iodide did not induce release of previously bound iron but did interfere with the ability of unsaturated transferrin to subsequently form a stable iron complex. Similarly, Winterbourn and Malloy (38) noted that both ferrilactoferrin and ferritransferrin did not release iron to any great extent upon exposure to either $\mathrm{HOCl}$ or a $\mathrm{MPO} / \mathrm{H}_{2} \mathrm{O}_{2} / \mathrm{Cl}^{-}$system. Exposure of the apo forms of these proteins to either system decreased the subsequent ability of lactoferrin and transferrin to bind iron. However, lactoferrin was much more resistant to inactivation.

In contrast to our data, Halliwell and colleagues reported (42) that exposure of either apotransferrin or apolactoferrin to $\mathrm{HOCl}$ had no effect on the ability of these proteins to limit formation of hydroxyl radical generated by iron-dependent hydroxyl radical generating systems. Specifics of the $\mathrm{HOCl}$ incubation are not described in their report making direct comparison of their results and ours difficult. Of interest, we too failed to note an effect of $\mathrm{HOCl}$ on apotransferrin if bicarbonate ion was present during the period of $\mathrm{HOCl}$ exposure. Clark and Pearson found (39) evidence that amino acids (particularly tyrosine) near the iron-binding sites of transferrin may be uniquely sensitive to MPO-mediated iodination. Bicarbonate is felt to bind in close proximity to the iron binding site of transferrin, most likely to an arginine residue $(13,67,68)$.
There is evidence that bicarbonate binding to some types of transferrins may alter protein conformation (69). It seems possible that such a process could decrease the susceptibility of the iron binding site to halogination, contributing to the protective effects of bicarbonate on $\mathrm{HOCl}$ induced decreases in apotransferrin iron-binding capacity.

Pseudomonas and other bacterial infections frequently begin at mucosal surfaces where their secretory products contribute to an array of proteases already present. The arrival of neutrophils with their release of granule proteases and generation of MPO-derived oxidants would provide further potential for inactivation of local iron-binding proteins. Thus, it is perhaps not surprising that lactoferrin whose role it is to function in this milieu of oxidants and proteases is more resistant to damage than transferrin which serves primarily as an iron transport protein in serum, a much less hostile environment.

Nevertheless, transferrin does contribute to the iron-binding potential at mucosal sites such as the pulmonary airways (54) where $P$. aeruginosa is an important pathogen. $P$. aeruginosa pulmonary infections are highly destructive and have an associated mortality approaching $70 \%$ even with appropriate antibiotic intervention (24). In addition, $P$. aeruginosa colonization/infection of the airways of patients with cystic fibrosis is temporally associated with the onset of the progressive deterioration of lung function which eventually is responsible for the death of $>90 \%$ of these individuals (24). A wide variety of $P$. aeruginosa-derived enzymes including pseudomonas elastase have been implicated as virulence factors in infections with this organism (24-27). Other products such as bacterial siderophores have also been implicated (24). We have recently shown that iron bound to the $P$. aeruginosa siderophore pyochelin can function as a hydroxyl radical catalyst possibly leading to local tissue injury (49). It seems possible that alterations of ferrictransferrin products by pseudomonas elastase or neutrophilderived MPO could contribute to some of the local tissue injury associated with $P$. aeruginosa infections.

\section{Acknowledgments}

We wish to thank Drs. Charles Cox, Robert Fick, Jr., and Robert Clark for their gifts of reagents and helpful suggestions, Michelle Railsback and Tedmund Roeder for technical assistance, and Naomi Erickson for her help with preparation of the manuscript.

This work was supported through the Veterans Administration Research Service, Public Health Service grants HL 44275 and AI 28412, the Cystic Fibrosis Foundation, the Pfizer Scholars Program, and the Sandoz Foundation for Gerontologic Research. It was performed during the tenure of B. E. Britigan as a Veterans Administration Research Associate.

\section{References}

1. Root, R. K., and M. S. Cohen. 1981. The microbicidal mechanisms of human neutrophils and eosinophils. Rev. Infect. Dis. 3:565-598.

2. Weiss, S. J. 1986. Oxygen, ischemia and inflammation. Acta Physiol. Scand. 548(Suppl.):9-37.

3. Klebanoff, S. J., and C. B. Hamon. 1972. Role of myeloperoxidase-mediated antimicrobial systems in intact leukocytes. J. Reticuloendothel. Soc. 12:170-196.

4. Weiss, S. J., M. D. Lampert, and S. T. Test. 1983. Long-lived oxidants generated by human neutrophils: characterization and bioactivity. Science (Wash. DC). 222:625-628.

5. Britigan, B. E. G. M. Rosen, Y. Chai, and M. S. Cohen. 1986. Do human neutrophils make hydroxyl radical? Detection of free radicals generated by human neutrophils activated with a soluble or particulate stimulus using electron paramagnetic resonance spectrometry. J. Biol. Chem. 261:4426-4431. 
6. Winterbourn, C. C. 1986. Myeloperoxidase as an effective inhibitor of hydroxyl radical production: implications for the oxidative reactions of neutrophils. J. Clin. Invest. 78:545-550.

7. Thomas, M. J., P. S. Shirley, C. Hedrick, and L. R. DeChatelet. 1986. Role of free radical processes in stimulated human polymorphonuclear leukocytes. Biochemistry. 25:8042-8048.

8. Kaur, H., Z. Fagerheim, M. Grootveld, A. Puppo, and B. Halliwell. 1988. Aromatic hydroxylation of phenylalanine as an assay for hydroxyl radicals: application to activated neutrophils and heme protein leghemoglobin. Anal. Biochem. 172:360-367.

9. Greenwald, R. A., S. W. Rush, S. A. Mark, and Z. Weitz. 1989. Conversion of superoxide generated by polymorphonuclear leukocytes to hydroxyl radical: a direct spectrophotometric detection system based on degradation of deoxyribose. Free Radical Biol. \& Med. 6:385-392.

10. Pou, S., M. S. Cohen, B. E. Britigan, and G. M. Rosen. 1989. Spin trapping and human neutrophils: limits of detection of hydroxyl radical. J. Biol. Chem. 264:12299-12302.

11. Britigan, B. E., T. J. Coffman, and G. R. Buettner. 1990. Spin trap evidence for the lack of significant hydroxyl radical production during the respiration burst of human phagocytes using a spin adduct resistant to superoxide mediated destruction. J. Biol. Chem. 265:2650-2656.

12. Bullen, J. J., H. J. Rogers, and E. Griffiths. 1978. Role of iron in bacterial infection. Curr. Top. Microbiol. Immunol. 80:1-35.

13. Legrand, D., J. Mazurier, J. Montreuil, and G. Spik. 1988. Structure and spatial conformation of the iron-binding sites of transferrins. Biochimie. 70:1185-1195.

14. Masson, P. L., J. F. Heremans, and C. H. Dive. 1966. Studies on lactoferrin, an iron-binding protein common to many external secretions. Clin. Chim. Acta. 14:735-739.

15. Ambruso, D. R., and R. B. Johnston, Jr. 1981. Lactoferrin enhances hydroxyl radical production by human neutrophils, neutrophil particulate fractions and an enzymatic generating system. J. Clin. Invest. 67:352-360.

16. Bannister, J. V., W. H. Bannister, H. A. O. Hill, and P. J. Thornalley. 1982. Enhanced production of hydroxyl radicals by the xanthine-xanthine oxidase reaction in the presence of lactoferrin. Biochim. Biophys. Acta. 715:116120.

17. Motohasi, N., and I. Mori. 1983. Superoxide dependent formation of hydroxyl radical catalyzed by transferrin. FEBS (Fed. Eur. Biochem. Soc.) Lett. 157:197-199.

18. Aruoma, O. I., and B. Halliwell. 1987. Superoxide-dependent and ascorbate-dependent formation of hydroxyl radicals from hydrogen peroxide in the presence of iron are lactoferrin and transferrin promoters of hydroxyl-radical generation? Biochem. J. 241:273-278.

19. Winterbourn, C. C. 1983. Lactoferrin-catalyzed hydroxyl radical production: additional requirements for a chelating agent. Biochem. J. 210:15-19.

20. Baldwin, D. A., E. R. Jenny, and P. Aisen. 1984. The effect of human serum transferrin and milk lactoferrin on hydroxyl radical formation from superoxide and hydrogen peroxide. J. Biol. Chem. 259:13391-13394.

21. Buettner, G. R. 1987. The reaction of superoxide, formate radical, and hydrated electron with transferrin and its model compound, Fe(III)-ethylenediamine-N, $N^{\prime}$-bis [2-(2-hydroxyphenyl) acetic acid] as studied by pulse radiolysis. $J$. Biol. Chem. 262:11995-11998.

22. Gutteridge, J. M. C., S. K. Paterson, A. W. Segal, and B. Halliwell. 1981. Inhibition of lipid peroxidation by the iron-binding protein lactoferrin. Biochem. J. 199:259-261.

23. Ward, P. A., G. O. Till, R. Kunkel, and C. Beauchamp. 1983. Evidence for the role of hydroxyl radical in complement and neutrophil-dependent tissue injury. J. Clin. Invest. 72:789-801.

24. Fick, R. B., Jr. 1989. Pathogenesis of the Pseudomonas lung lesion in cystic fibrosis. Chest. 96:158-164.

25. Pier, G. B. 1985. Pulmonary disease associated with Pseudomonas aeruginosa in cystic fibrosis: current status of the host bacterium interaction. $J$. Infect. Dis. 151:575-580.

26. Suter, S., O. B. Schaad, L. Roux, U. E. Nydegger, and F. A. Waldvogel 1984. Granulocyte neutral proteases and Pseudomonas elastase as possible causes of airway damage in patients with cystic fibrosis. J. Infect. Dis. 149:523-531.

27. Dunn, M. M., M. Dunne, and D. W. Kamp. 1990. Polymorphonuclear leukocyte- and Pseudomonas aeruginosa-induced damage to a human pulmonary epithelial cell line. J. Infect. Dis. 162:172-177.

28. Mohammed, J. R., B. S. Mohammed, L. J. Pawluk, D. M. Bucci, and W. B. Davis. 1988. Purification and cytotoxic potential of myeloperoxidase in cystic fibrosis sputum. J. Lab. Clin. Med. 112:711-720.

29. Holder, I. A. 1983. Experimental studies of the pathogenesis of infections due to Pseudomonas aeruginosa: effect of treatment with protease inhibitors. Rev. Infect. Dis. 5:S914-S921.

30. Kreger, A. S. 1983. Pathogenesis of Pseudomonas aeruginosa ocular dis ease. Rev. Infect. Dis. 5:S931-S935.

31. Wretlind, B., and O. R. Pavlovskis. 1983. Pseudomonas elastase and its role in pseudomonas infections. Rev. Infect. Dis. 5:S998-S1004.
32. Doring, G., M. Pfestorf, K. Botzenhart, and M. A. Abdallah. 1988. Impact of proteases on iron uptake of Pseudomonas aeruginosa pyoverdin from transferrin and lactoferrin. Infect. Immun. 56:291-293.

33. Brines, R. D., and J. H. Brock. 1983. The effect of trypsin and chymotrypsin on the in vitro antimicrobial iron-binding properties of lactoferrin in human milk and bovine colostrom. Biochim. Biophys. Acta. 759:229-235.

34. Line, W. F., D. A. Sly, and A. Bezkorovainy. 1976. Limited cleavage of human lactoferrin with pepsin. Int. J. Biochem. 9:203-208.

35. Bluard-Deconinck, J.-M., J. Williams, R. W. Evans, J. van Snick, P. A. Osinski, and P. L. Masson. 1978. Iron-binding fragments from the $\mathbf{N}$-terminal and C-terminal regions of human lactoferrin. Biochem. J. 171:321-327.

36. Evans, R. W., and J. Williams. 1978. Studies of the binding of different iron donors to human serum transferrin and isolation of iron-binding fragments from the N-and C-terminal regions of the protein. Biochem. J. 173:543-552.

37. Esparza, I., and J. H. Brock. 1980. The effect of trypsin digestion on the structure and iron-donating properties of transferrins from several species. Biochim. Biophys. Acta. 622:297-307.

38. Winterbourn, C. C., and A. L. Malloy. 1988. Susceptibilities of lactoferrin and transferrin to myeloperoxidase-dependent loss of iron-binding capacity. Bio chem. J. 250:613-616.

39. Clark, R. A., and D. W. Pearson. 1989. Inactivation of transferrin iron binding capacity by the neutrophil myeloperoxidase system. J. Biol. Chem. 264:9420-9427.

40. Penner, M. H., R. B. Yamasaki, D. T. Osuga, D. R. Babin, C. F. Meares, and $R$. E. Feeney. 1983. Comparative oxidations of tyrosines and methionines in transferrins: human serum transferrin, human lactotransferrin, and chicken ovotransferrin. Arch. Biochem. Biophys. 225:740-747.

41. Geoghegan, K. F., J. L. Dallas, and R. E. Feeney. 1980. Periodate inactivation of ovotransferrin and human serum transferrin. J. Biol. Chem. 242:2810 2815

42. Halliwell, B., O. I. Aruoma, M. Wasil, and J. M. C. Gutteridge. 1988. The resistance of transferrin, lactoferrin: caeruloplasm to oxidative damage. Biochem. J. 256:311-312.

43. Baldwin, D. A., and D. M. R. de Sousa. 1981. The effect of salts on the kinetics of iron release from N-and C-terminal monoferrictransferrins. Biochem. Biophys. Res. Commun. 99:1101-1107.

44. Bates, G. W., C. Billups, and P. Saltman. 1967. The kinetics and mechanism of iron (III) exchange between chelates and transferrin. I. The complexes of citrate and nitrilotriacetic acid. J. Biol. Chem. 242:2810-2815.

45. Laemmli, U. K. 1970. Cleavage of structural proteins during the assemblage of the head of bacteriophage T4. Nature (Lond.). 227:680-685.

46. Wray, W., T. Boulikas, V. P. Wray, and R. Hancock. 1981. Silver stain of proteins in polyacrylamide gels. Anal. Biochem. 118:197-203.

47. Makey, D. G., and U. S. Seal. 1976. The detection of four molecular forms of human transferrin during the iron binding process. Biochim. Biophys. Acta. 453:250-256.

48. Nauseef, W. M. 1987. Posttranslational processing of a human myeloid lysosomal protein, myeloperoxidase. Blood. 70:1143-1150.

49. Coffman, T. J., C. D. Cox, B. L. Edeker, and B. E. Britigan. 1990. Possible role of bacterial siderophores in inflammation. Iron bound to the Pseudomonas siderophore pyochelin can function as a hydroxyl radical catalyst. J. Clin. Invest. 86:1030-1037.

50. Halliwell, B., and J. M. C. Gutteridge. 1981. Formation of a thiobarbituric acid-reactive substance from deoxyribose in the presence of iron salts. The role of superoxide and hydroxyl radicals. FEBS (Fed. Eur. Biochem. Soc.) Lett. 128:347-351.

51. Weiss, S. J. 1989. Tissue destruction by neutrophils. N. Engl. J. Med. 320:365-376.

52. Diguiseppi, J., and I. Fridovich. 1980. Ethylene from 2-keto-4-thiomethyl butyric acid: the Haber-Weiss reaction. Arch. Biochem. Biophys. 295:323-329.

53. Britigan, B. E., S. Pou, G. M. Rosen, D. M. Lilleg, and G. R. Buettner. 1990. Hydroxyl radical is not a product of the reaction of xanthine oxidase and xanthine. The confounding problem of adventitious iron bound to xanthine oxidase. J. Biol. Chem. 265:17533-17538.

54. Fick, R. B. Jr, G. P. Naegel, S. U. Squier, R. E. Wood, J. B. L. Gee, and H. Y. Reynolds. 1984. Proteins of the cystic fibrosis respiratory tract: fragmented immunoglobulin $\mathbf{G}$ opsonic antibody causing defective opsonophagocytosis. $J$. Clin. Invest. 74:236-248.

55. Bruce, M. C., L. Poncz, J. D. Klinger, R. C. Stern, J. Tomashefski, and D. G. Dearborn. 1985. Biochemical and pathological evidence for proteolytic destruction of lung connective tissue in the cystic fibrosis patient. Am. $R e v$. $R e$ spir. Dis. 132:529-535.

56. Suter, S., O. B. Schaad, H. Tegner, K. Ohlsson, D. Desgrandchamps, and F. A. Waldvogel. 1986. Levels of free granulocyte elastase in bronchial secretions from patients with cystic fibrosis. J. Infect. Dis. 153:902-909.

57. Goldstein, W., and G. Doring. 1986. Lysosomal enzymes from polymorphonuclear leukocytes and proteinase inhibitors in patients with cystic fibrosis. Am. Rev. Respir. Dis. 134:49-56.

58. Jackson, A. H., S. L. Hill, S. C. Afford, and R. A. Stockley. 1984. Sputum 
sol-phase proteases and elastase activity in patients with cystic fibrosis. Eur. $J$. Respir. Dis. 65:114-124.

59. Doring, G., A. Dalhoff, O. Vogel, H. Brunner, U. Droge, and K. Botzenhart. 1984. In vivo activity of proteases of Pseudomonas aeruginosa in a rat model. J. Infect. Dis. 149:532-537.

60. Williams, J., and K. Moreton. 1980. The distribution of iron between the metal binding sites of transferrin in human serum. Biochem. J. 185:483-488.

61. Leibman, A., and P. Aisen. 1979. Distribution of iron between the binding sites of transferrin in serum. Methods and results in normal human subjects. Blood. 53:1058-1065.

62. Zak, O., and P. Aisen. 1985. Iron is not randomly distributed between the binding sites of circulating human transferrin. In Proteins of Iron Storage and Transport. G. Spik, J. Montreuil, R. R. Crichton, and J. Mazurier, editors. Elsevier Science Publishers, New York. 61-64.

63. Britigan, B. E., G. M. Rosen, B. Y. Thompson, Y. Chai, and M. S. Cohen. 1986. Stimulated neutrophils limit iron-catalyzed hydroxyl radical formation as detected by spin trapping techniques. J. Biol. Chem. 261:17026-17032.

64. Britigan, B. E., D. J. Hassett, G. M. Rosen, D. R. Hamill, and M. S Cohen. 1989. Neutrophil degranulation inhibits potential hydroxyl radical for- mation: differential impact of myeloperoxidase and lactoferrin release on hydroxyl radical production by iron supplemented neutrophils assessed by spin trapping. Biochem. J. 264:447-455.

65. Reynolds, H. Y. 1987. Bronchoalveolar lavage. Am. Rev. Respir. Dis. 135:250-263.

66. Suter, S. 1989. The imbalance between granulocyte neutral proteases and antiproteases in bronchial secretions from patients with cystic fibrosis. Antibiot. Chemother. 42:158-168.

67. Zwier, J. L., J. B. Wooten, and J. S. Cohen. 1981. Studies of anion binding by transferrin using Carbon-13 nuclear magnetic resonance spectroscopy. Biochemistry. 20:3505-3510.

68. MacGillivray, R. T. A., E. Mendez, J. G. Shewale, S. K. Sinha, J. Lineback-Zins, and K. Brew. 1983. The primary structure of human serum transferrin. The structures of the seven cyanogen bromide fragements and the assembly of the complete structure. J. Biol. Chem. 258:3543-3553.

69. Oe, H., N. Takahasi, E. Doi, and M. Hirose. 1989. Effects of anion binding on the confirmations of the two domains of ovotransferrin. J. Biochem. 106 858-863. 